\title{
Electron Entanglement via a Quantum Dot
}

\author{
W. D. Oliver, F. Yamaguchi, and Y. Yamamoto \\ Quantum Entanglement Project, ICORP, JST, \\ E. L. Ginzton Laboratory, \\ Stanford University, Stanford, CA 94305
}

(Dated: July 15, 2001)

\begin{abstract}
This Letter presents a method of electron entanglement generation. The system under consideration is a single-level quantum dot with one input and two output leads. The leads are arranged such that the dot is empty, single electron tunneling is suppressed by energy conservation, and two-electron virtual co-tunneling is allowed. This yields a pure, non-local spin-singlet state at the output leads. Coulomb interaction is the nonlinearity essential for entanglement generation, and, in its absence, the singlet state vanishes. This type of electron entanglement is a four-wave mixing process analogous to the photon entanglement generated by a $\chi^{(3)}$ parametric amplifier.
\end{abstract}

Identical quantum particles are inherently indistinguishable, and this oftentimes leads to non-classical behavior such as entanglement in quantum mechanical systems. The Einstein-Podolsky-Rosen (EPR) state [1, 2, 3] is an interesting example of two-particle entanglement, because it has potential use in secure quantum communication protocol [4. 5], quantum information processing [6], and fundamental tests of quantum mechanics [7]. Photons in nonlinear media interact to produce polarization-entangled EPR pairs and have been used in experimental demonstrations of quantum state teleportation [8, 9, 10], quantum non-demolition measurements [11], and violations of Bell's inequality [12, 13]. Although entanglement with ions 14 and between atoms and cavity field modes has been demonstrated [15, 16, 17, to our knowledge, there have yet to be any experimental demonstrations specifically utilizing EPR pair-type entangled electrons. Recently, there have been several proposals to generate 18, 19, 20, 21] and detect [22, 23, 24, 25] entangled electrons. Electrons have been demonstrated to have long spin dephasing times in semiconductors [26, 27]. In addition, the quantum optics tools [28, 29], for example an electron waveguide 30, 31], beamsplitter [32, 33, intensity interferometer [33, 34], and collision analyzer [24, 32, required to detect entangled electrons have been demonstrated in two-dimensional electron gas systems. Furthermore, the lossless nature of electrons and the noiseless property of a cryogenic Fermi source may provide experimental advantages, for example high detection efficiency [14, 25], over their photon counterparts.

In this Letter, we consider a means to generate entangled EPR pairs with electrons using a three-port quantum dot (Fig. 17a) operating in the coherent tunneling regime [35, 36]. The dot consists of a single input lead and two output leads, with an energy band diagram

\footnotetext{
*woliver@stanford.edu; URL : f eynman. stanford .edu

† also at NTT Basic Research Laboratories, 3-1 MorinosatoWakamiya Atsugi, Kanagawa, 243-01 Japan
}
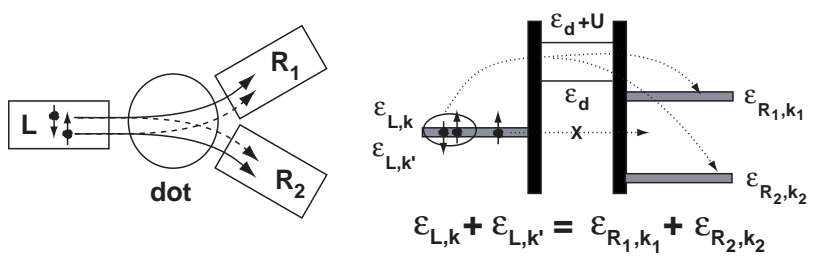

FIG. 1: a) Three-port quantum dot. b) Energy band diagram for three-port quantum dot with non-degenerate energy leads which act as energy filters.

shown in Fig. 17b. The lead arrangement is such that the dot is empty. Conceptually, there are two key factors to the successful operation of this entangler. The first is that the leads are non-degenerate and of narrow width in energy, thus acting as "energy filters". Single electron tunneling does not conserve energy and is forbidden. However, the lead energies can be arranged such that two-electron co-tunneling events do conserve energy, and thus the lowest order contribution to the tunneling current is two-electron virtual co-tunneling through the dot. The second is that double occupancy of the dot incurs a charging energy. The Coulomb interaction mediates electron entanglement in this system, as a nonlinear medium does for photon entanglement. In its absence, the singlet state generation vanishes. Fundamentally, this is because the system has a high degree of symmetry. With the Coulomb interaction turned off, the singlet and triplet states destructively interfere. However, in the presence of Coulomb interaction, the system symmetry is reduced and the singlet state destructive interference becomes imperfect, while the triplet state destructive interference remains complete. This leads to a net singlet state amplitude at the output of the dot.

Experimental verification of electron entanglement might be achieved through an electron bunching/antibunching experiment [23, 24], or spin correlation measurements and, ultimately, a Bell's inequality test [25. Since, to our knowledge, there is no demonstrated method to coincidence count with electrons, an entan- 
gler ideally should have high efficiency. That is, the output is a continuous and coherent singlet state with no noise. Then, at least in principle, standard noise measurement techniques could be adopted to infer the degree and type of entangled state produced. Although outside the scope of this Letter, we consider elsewhere the issue of resonance conditions for the quantum dot entangler to enhance detection [37].

The quantum dot is analyzed in the coherent tunneling regime using the Anderson Hamiltonian with an onsite Coulomb energy term U. We consider only a single, spin-degenerate energy level for the dot, and there are no single electron excitations within the dot.

$$
\begin{aligned}
\hat{H}_{\text {And }}= & \sum_{\eta, k, \sigma} \varepsilon_{\eta, k} \hat{a}_{\eta, k, \sigma}^{\dagger} \hat{a}_{\eta, k, \sigma}+\sum_{\sigma} \varepsilon_{d} \hat{c}_{\sigma}^{\dagger} \hat{c}_{\sigma} \\
& +U \hat{n}_{\uparrow} \hat{n}_{\downarrow}+\sum_{\eta, k, \sigma}\left(V_{\eta} \hat{a}_{\eta, k, \sigma}^{\dagger} \hat{c}_{\sigma}+\text { h.c. }\right)
\end{aligned}
$$

where $\eta \in\left\{L, R_{1}, R_{2}\right\}$ is the lead label, $k$ is the lead electron momentum, $\sigma \in\{\uparrow, \downarrow\}$ is the electron spin, $V_{\eta}$ is the overlap matrix element between the dot and the lead states, $\hat{a}\left(\hat{a}^{\dagger}\right)$ is the annihilation (creation) operator for the lead electrons, $\hat{c}\left(\hat{c}^{\dagger}\right)$ is the annihilation (creation) operator for the dot electrons, and $\hat{n}_{\sigma} \equiv \hat{c}_{\sigma}^{\dagger} \hat{c}_{\sigma}$ is the dot electron number operator. The dot energy levels, $\varepsilon_{d}$ and $\varepsilon_{d}+U$, in Fig. 目 are taken to be off resonance with the leads. The left lead energy is below its quasi-Fermi level so that the lead is full of electrons. The right leads are empty. In addition, we set $\varepsilon_{d}=0$, thereby referencing all energies to $\varepsilon_{d}$. The lead energies $\varepsilon_{\eta, k}$ and the charging energy $U$ are left as parameters which can be adjusted to consider different dot configurations.

In all cases, the three-port quantum dot is biased such that single electron tunneling from the left lead to the right lead is suppressed, that is, $\varepsilon_{L, k} \neq \varepsilon_{R_{1}, k_{1}} \neq \varepsilon_{R_{2}, k_{2}}$. The lead energy level widths are narrow enough that energy overlap between different leads does not occur. However, two-electron virtual co-tunneling does conserve energy, that is, $\varepsilon_{L, k}+\varepsilon_{L, k^{\prime}}=\varepsilon_{R_{1}, k_{1}}+\varepsilon_{R_{2}, k_{2}}$, requiring one electron from the left lead to go to lead $R_{1}$ and the other to go to lead $R_{2}$. This is the energy conserving process considered throughout this Letter.

In terms of a perturbation expansion in the tunneling matrix element $V$, the lowest order contribution to the current from the left lead to the right leads is $\mathcal{O}\left(V^{4}\right)$. Given the assumption that only one dot energy level is relevant in this quantum dot, all higher-order terms contribute to either the self-energy of the electrons or are higher orders of two-electron co-tunneling. Therefore, a two-electron initial state is used in this model,

$$
\left|\phi_{i}\right\rangle=\hat{a}_{L, k, \sigma^{\prime}}^{\dagger} \hat{a}_{L, k^{\prime}, \sigma^{\prime}}^{\dagger}|0\rangle
$$

where $|0\rangle$ is the zero-particle state of this model system. This initial state is an arbitrary selection of two electrons from the entire left lead $T \approx 0$ ground state. Physical quantities, such as current, can be found by summing over all possible two-electron input and output states.

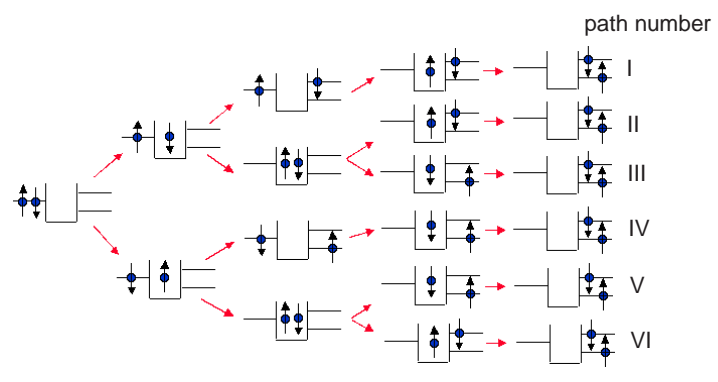

FIG. 2: Multiple paths by which two electrons can virtually co-tunnel from the left lead through an empty dot to the two right leads.

We introduce the notation $E_{L}, \Delta_{L}$, and $\Delta_{R}$ to parameterize $\varepsilon_{L, k}, \varepsilon_{L, k^{\prime}}, \varepsilon_{R_{1}}$, and $\varepsilon_{R_{1}}$ and simplify the presentation.

$$
\begin{aligned}
E_{L} & \equiv \frac{1}{2}\left(\varepsilon_{L, k}+\varepsilon_{L, k^{\prime}}\right)=\frac{1}{2}\left(\varepsilon_{R_{1}, k_{1}}+\varepsilon_{R_{2}, k_{2}}\right) \\
\Delta_{L} & \equiv \frac{1}{2}\left(\varepsilon_{L, k}-\varepsilon_{L, k^{\prime}}\right) \\
\Delta_{R} & \equiv \frac{1}{2}\left(\varepsilon_{R_{1}, k_{1}}-\varepsilon_{R_{2}, k_{2}}\right)
\end{aligned}
$$

The two-electron initial state has energies $\varepsilon_{L, k}, \varepsilon_{L, k^{\prime}}=$ $E_{L} \pm \Delta_{L}$ within the left lead, and the two-electron final state has energies $\varepsilon_{R_{1}, k_{1}}, \varepsilon_{R_{2}, k_{2}}=E_{L} \pm \Delta_{R}$ in the right leads. The energy widths of the leads are denoted as $\delta_{L}$ for the left lead and $\delta_{R}$ for each of the two right leads. Suppressing single electron tunneling requires that $\Delta_{L}<$ $\Delta_{R}$ for any $\Delta_{L}$ and $\Delta_{R}$. In practice, this would mean that $\delta_{L}, \delta_{R}<<\Delta_{R}$.

We consider first the case of a spin-up and spin-down electron tunneling through the dot to the output leads as indicated in the Fig. 1. The initial state is Eqn. 2 with $\sigma=\uparrow$ and $\sigma^{\prime}=\downarrow$. The time-ordering operator in the perturbative expansion 33, 39] leads to six paths comprising twelve unique time-orderings by which these two electrons can virtually co-tunnel through the quantum dot. Each path has two time-orderings, one as shown in Fig. 2 and another due to the exchange of the output leads $R_{1}$ and $R_{2}$. This interchange of $R_{1}$ and $R_{2}$ introduces a minus sign due to the electron commutation relation when the state is written in its "normal ordering" form. Due to the coherent nature of the virtual tunneling process, the six paths will interfere to produce the resulting final state output. The six paths are presented in terms of a singlet and a triplet contribution using the shorthand notation $|S\rangle,|T\rangle \equiv\left(\hat{a}_{R_{1} \uparrow}^{\dagger} \hat{a}_{R_{2} \downarrow}^{\dagger} \mp \hat{a}_{R_{1} \downarrow}^{\dagger} \hat{a}_{R_{2} \uparrow}^{\dagger}\right)|0\rangle$ to indicate the singlet and triplet states. The factor $C \equiv$ $V_{L}^{* 2} V_{R_{1}} V_{R_{2}} \frac{\exp \left[-\frac{i}{\hbar}\left(2 E_{L}-\varepsilon_{R_{1}, k_{1}}-\varepsilon_{R_{2}, k_{2}}\right)\right]}{\left(2 E_{L}-\varepsilon_{R_{1}, k_{1}}-\varepsilon_{R_{2}, k_{2}}\right)}$ is common to all terms and omitted until the end.

$$
\left|\phi_{I}\right\rangle=\frac{\left(\Delta_{R}^{2}-\Delta_{L} E_{L}\right)|S\rangle-\Delta_{R}\left(E_{L}-\Delta_{L}\right)|T\rangle}{\left(E_{L}-\Delta_{L}\right)\left(E_{L}^{2}-\Delta_{R}^{2}\right)\left(\Delta_{L}^{2}-\Delta_{R}^{2}\right)}
$$




$$
\begin{aligned}
\left|\phi_{I I}\right\rangle & =\frac{E_{L}|S\rangle-\Delta_{R}\left(E_{L}-\Delta_{L}\right)|T\rangle}{\left(E_{L}-\Delta_{L}\right)\left(E_{L}^{2}-\Delta_{R}^{2}\right)\left(2 E_{L}-U\right)} \\
\left|\phi_{I I I}\right\rangle & =\frac{E_{L}|S\rangle+\Delta_{R}\left(E_{L}-\Delta_{L}\right)|T\rangle}{\left(E_{L}-\Delta_{L}\right)\left(E_{L}^{2}-\Delta_{R}^{2}\right)\left(2 E_{L}-U\right)} \\
\left|\phi_{I V}\right\rangle & =\frac{\left(\Delta_{R}^{2}+\Delta_{L} E_{L}\right)|S\rangle+\Delta_{R}\left(E_{L}+\Delta_{L}\right)|T\rangle}{\left(E_{L}+\Delta_{L}\right)\left(E_{L}^{2}-\Delta_{R}^{2}\right)\left(\Delta_{L}^{2}-\Delta_{R}^{2}\right)} \\
\left|\phi_{V}\right\rangle & =\frac{E_{L}|S\rangle+\Delta_{R}\left(E_{L}+\Delta_{L}\right)|T\rangle}{\left(E_{L}+\Delta_{L}\right)\left(E_{L}^{2}-\Delta_{R}^{2}\right)\left(2 E_{L}-U\right)} \\
\left|\phi_{V I}\right\rangle & =\frac{E_{L}|S\rangle-\Delta_{R}\left(E_{L}+\Delta_{L}\right)|T\rangle}{\left(E_{L}+\Delta_{L}\right)\left(E_{L}^{2}-\Delta_{R}^{2}\right)\left(2 E_{L}-U\right)}
\end{aligned}
$$

The output states from paths $I$ and $I V$ do not incur a charging energy $U$, while the remaining states do incur a charging energy $U$ during the virtual tunneling event.

It is now clear how the triplet states destructively in- terfere. Paths $I$ and $I V$, paths $I I$ and $I I I$, and paths $V$ and $V I$ have triplet contributions which cancel. Only singlet states remain, and the singlet contributions along paths $I I$ and $I I I$ and along paths $V$ and $V I$ are identical. Furthermore, paths $I, I I$, and $I I I$ contain the factor $\left(E_{L}-\Delta_{L}\right)$ in the denominator, indicating that the first electron to enter the dot along these paths was the $L, k$ electron. Conversely, paths $I V, V$, and $V I$ contain the factor $\left(E_{L}+\Delta_{L}\right)$ in the denominator, indicating that the first electron to enter was the $L, k^{\prime}$ electron. Combining paths according to the electron which initially enters the dot yields the following expressions. The triplet state contributions of paths $I$ and $I V$ are simply written as $\pm \tau\left(E_{L}, \Delta_{L}, \Delta_{R}\right)$, since they cancel upon summation as stated above.

$$
\begin{aligned}
\left|\phi_{I}\right\rangle+\left|\phi_{I I}\right\rangle+\left|\phi_{I I I}\right\rangle & =\frac{-U\left(\Delta_{R}^{2}-\Delta_{L} E_{L}\right)-2 \Delta_{L} E_{L}\left(E_{L}-\Delta_{L}\right)}{\left(E_{L}-\Delta_{L}\right)\left(\Delta_{L}^{2}-\Delta_{R}^{2}\right)\left(E_{L}^{2}-\Delta_{R}^{2}\right)\left(2 E_{L}-U\right)}|S\rangle+\tau\left(E_{L}, \Delta_{L}, \Delta_{R}\right)|T\rangle \\
\left|\phi_{I V}\right\rangle+\left|\phi_{V}\right\rangle+\left|\phi_{V I}\right\rangle & =\frac{-U\left(\Delta_{R}^{2}+\Delta_{L} E_{L}\right)+2 \Delta_{L} E_{L}\left(E_{L}+\Delta_{L}\right)}{\left(E_{L}+\Delta_{L}\right)\left(\Delta_{L}^{2}-\Delta_{R}^{2}\right)\left(E_{L}^{2}-\Delta_{R}^{2}\right)\left(2 E_{L}-U\right)}|S\rangle-\tau\left(E_{L}, \Delta_{L}, \Delta_{R}\right)|T\rangle
\end{aligned}
$$

The interference of the singlet states is now clear. In the absence of the charging energy $U$, the singlet states in paths $I, I I$, and $I I I$ destructively and completely interfere with those in paths $I V, V$, and $V I$. However, the presence of the charging energy $U$ adds an additional contribution to each set of paths. This additional $U$ dependent contribution contains a part which still destructively interferes between the two sets of paths, but it also contains a part which constructively interferes, leaving a residual singlet state at the output. Reinserting the common factor $C$, the final result for the total output state is

$$
|\psi(t)\rangle=\frac{2 C E_{L}}{\left(E_{L}^{2}-\Delta_{R}^{2}\right)\left(E_{L}^{2}-\Delta_{L}^{2}\right)} \frac{U}{2 E_{L}-U}|S\rangle .
$$

The remaining case is the virtual co-tunneling of two electrons with the same spin. The input state is Eqn. 2 with $\sigma=\sigma^{\prime}$. Due to the Pauli exclusion principle, only paths $I$ and $I V$ can contribute to the output state amplitude. Since there are no singlet states in the same spin case and the triplet states destructively interfere for paths $I$ and $I V$, there is no same-spin co-tunneling contribution at the output.

The conclusion is that virtual co-tunneling through an empty quantum dot in the presence of Coulomb interaction generates entangled electron spin-singlet states. The physical meaning of equation 14 can be explained in the following way. Two electrons (with possibly different energies) virtually tunnel through the quantum dot along six different paths (twelve unique time-orderings).
These different paths interfere with each other. Two of the paths $(I$ and $I V)$ do not include double occupancy of the dot and, thus, incur no charging energy. The remaining four paths do incur a charging energy due to a virtual double occupancy of the dot. The interference for the triplet states is destructive and complete. That is, the triplet state contribution of each path has an oppositeparity partner with which it destructively interferes. The result is independent of the charging energy $U$, since the triplet states of those paths which do depend on charging energy destructively self-interfere, and those paths which do not self-interfere also. There is no requirement to "mix" the $U$-dependent and $U$-independent paths to get the complete destructive interference of the triplet states.

However, the system symmetry or "non-mixing property" is broken in the case of the singlet states. The interference is destructive for the singlet states, but only complete when the charging energy $U=0$. For $U \neq 0$, the destructive interference becomes imperfect, and residual singlet state remains at the output. One perspective is that the interference occurs between paths which share the same electron in the first tunneling event. For example, there are three paths which share the $L, k$ electron first tunneling into the dot. Call the $L, k$ electron paths set $A$ and the remaining three $L, k^{\prime}$ electron paths set $B$. Within each set, two of the three paths depend on $U$ and one does not. Therefore, within each set, there is a mixing of paths which do depend and do not depend on $U$. In the limit $U=0$, the contributions of set $\mathrm{A}$ 
and set B are equal in magnitude and of opposite parity; they cancel when summed. However, $U \neq 0$ breaks this symmetry, creating a residual $U$-dependent contribution that does not cancel when sets A and B are summed. Admittedly, the choice of grouping paths into sets $\mathrm{A}$ and $\mathrm{B}$, while physically compelling, is nonetheless arbitrary. It may be more appropriate to state that $U$ breaks the system symmetry, leading to a net singlet contribution.

The physical meaning of the same-spin case is similar. The same-spin electrons can only form a spin-triplet state at the output and, therefore, destructively interfere completely. Furthermore, the same-spin case only traverses the dot along paths $I$ and $I V$, which do not incur any charging energy. In this sense, there is no non-linear interaction to create an entangled state at the output, and so, from this perspective also, the destructive interference is complete.

The nonlinearity in this system, the Coulomb interac- tion as manifest in the charging energy $U$, is necessary to observe entanglement at the output. The optical analogue to this system is a $\chi^{(3)}$ parametric amplifier with a vacuum state as its input. Two input photons, created through spontaneous emission, interact within the $\chi^{(3)}$ nonlinear medium and generate an entangled pair of output photons. This is a four-wave mixing process; two photons in and two photons out. The $\chi^{(3)}$ nonlinearity mediates the entanglement in the photon case, whereas it is the Coulomb interaction in the electron case. This correspondence between nonlinear optics and Coulombmediated wave-mixing may be applicable to other quantum dot configurations and other electron or composite particle systems that exhibit Coulomb charging behavior.

The authors gratefully acknowledge support from MURI and JSEP. W. D. O. gratefully acknowledges additional support from MURI and the NDSEG Fellowship Program.
[1] A. Einstein et al., Phys. Rev. 47, 777 (1935).

[2] N. Bohr and L. Rosenfeld, Phys. Rev. 48, 669 (1938).

[3] D. Bohm, Quantum Theory, Constable, London (1954).

[4] C. H. Bennett and G. Brassard, Proceedings of the IEEE Converence on Computers, Systems, and Signal Processing, Bangalore, India (IEEE, New York, 1984) 174 (1984).

[5] A. K. Ekert, Phys. Rev. Lett. 67, 661 (1991).

[6] C. H. Bennett and D. P. DiVincenzo, Nature 404, 247 (2000).

[7] J. S. Bell, Physics 1, 195 (1964).

[8] D. Bouwmeester et al., Nature 390, 575 (1997).

[9] D. Boschi et al., Phys. Rev. Lett. 80, 1121 (1998).

[10] A. Furusawa et al., Science 282, 706 (1998).

[11] P. Grangier et al., Nature 396, 537 (1998).

[12] A. Aspect et al., Phys. Rev. Lett. 47, 460 (1981).

[13] G. Weihs et al., Phys. Rev. Lett. 81, 5039 (1998).

[14] M. A. Rowe et al., Nature 409, 794 (2001).

[15] E. Hagley et al., Phys. Rev. Lett. 79, 1 (1997).

[16] X. Maitre et al., Phys. Rev. Lett. 79, 769 (1997).

[17] G. Nogues et al., Nature 400, 239 (1999).

[18] D. Loss and D. P. DiVincenzo, Phys. Rev. A 57, 120 (1998).

[19] G. Burkard et al., Phys. Rev. B 59, 2070 (1999).

[20] C. H. W. Barnes et al. Phys. Rev. B 62, 8410 (2000)

[21] P. Recher et al., Phys. Rev. B 63, 165314 (2001).

[22] D. Loss and E. V. Sukhorukov Phys. Rev. Lett. 84, 1035 (2000).
[23] G. Burkard et al., Phys. Rev. B 61, R16303 (2000).

[24] W. D. Oliver et al. in Quantum Mesoscopic Phenomena and Mesoscopic Devices in Microelectronics, I. O. Kulik and R. Ellialtioğlu ed., Kluwer Academic Publishers, Dordrecht (2000).

[25] X. Maître et al., Physica E 6, 301 (2000).

[26] J. M. Kikkawa et al., Science 277, 1284 (1997).

[27] J. M. Kikkawa and D. D. Awschalom et al., Nature 397, 139 (1999).

[28] M. Büttiker, Phys. Rev. B 54, 12485 (1992).

[29] T. Martin and R. Landauer, Phys. Rev. B 45, 1742 (1992).

[30] B. J. van Wees et al., Phys. Rev. Lett. 60, 848 (1988).

[31] D. A. Wharam et al., J. Phys. C 21, 209 (1988).

[32] R. C. Liu et al., Nature 391, 263 (1998).

[33] W. D. Oliver et al., Science 284, 299 (1999).

[34] M. Henny et al., Science 284, 296 (1999).

[35] D. V. Averin and Yu. V. Nazarov in Single Charge Tunneling H. Grabert and M. H. Devoret ed., NATO ASI Series B, Volume 294, Plenum Press, New York (1992).

[36] E. V. Sukhorukov et al., Phys. Rev. B 63, 125315 (2000).

[37] W. D. Oliver et al. in preparation.

[38] L. S. Rodberg and R. M. Thaler, Introduction to the Quantum Theory of Scattering, Academic Press, New York (1967).

[39] G. D. Mahan, Many Particle Physics, Plenum Press, New York (1990). 